\title{
Fucoidan modulates cytokine production and migration of THP-1-derived macrophages via colony-stimulating factor-1
}

\author{
PENG LI $^{1}$, HUAYANG WANG ${ }^{2}$, QIANQIAN SHAO ${ }^{2}$, BEIHUA KONG $^{1}$ and XUN QU ${ }^{2}$ \\ ${ }^{1}$ Department of Gynecology and Obstetrics; ${ }^{2}$ Institute of Basic Medical Sciences, \\ Qilu Hospital of Shandong University, Jinan, Shandong 250012, P.R. China
}

Received October 30, 2015; Accepted November 2, 2016

DOI: $10.3892 / \mathrm{mmr} .2017 .6228$

\begin{abstract}
Fucoidan is known for its various biological activities, including immunomodulatory effects on immune cells. However, the effect of fucoidan on the functions of macrophages remains to be elucidated. The present study examined the effects of fucoidan on cytokine production and migration of THP-1-derived macrophages and its potential mechanisms. Fucoidan was added during the differentiation process of THP-1-derived macrophages along with lipopolysaccharide and interferon- $\gamma$ for $42 \mathrm{~h}$, and then macrophages were harvested for functional assays. Fucoidan altered the morphology of THP-1-derived macrophages, and also attenuated their migration activity and pro-inflammatory cytokine production. Additionally, THP-1-derived macrophages intensively produced colony-stimulating factor-1 (CSF-1), which was significantly decreased by fucoidan. CSF-1 neutralizing antibody attenuated the basic production level of pro-inflammatory cytokines in macrophages. Furthermore, when recombinant human CSF-1 was added along with fucoidan, the attenuating effects of fucoidan on migration and cytokine production were significantly reversed. In conclusion, the present study suggests that macrophages appear to be a potential target in the immunomodulatory action of fucoidan, and CSF-1 may be involved in this modulation.
\end{abstract}

\section{Introduction}

Fucoidan is a series of sulfated polysaccharides derived from marine brown algae, predominantly composed of sulfate and

Correspondence to: Dr Xun Qu, Institute of Basic Medical Sciences, Qilu Hospital of Shandong University, 107 Wenhuaxi Road, Jinan, Shandong 250012, P.R. China

E-mail: quxun@qiluhospital.com

Abbreviations: CSF-1, colony-stimulating factor-1; PMA, phorbol-12-myristate-13-acetate; Fu, fucoidan; NAb, neutralizing antibody

Key words: fucoidan, macrophages, cytokines, cell migration, colony-stimulating factor-1
L-fucose $(1,2)$. Numerous biological activities of fucoidan have been previously described, including anti-oxidant and antitumor properties, and roles in the regulation of metabolism and inflammatory modulation (3). Such properties make fucoidan a potential pharmaceutical and nutraceutical candidate for health control and disease prevention. The immunomodulatory effect is a primary property of fucoidan. Immune cells, including macrophages, natural killer cells, dendritic cells (DCs) and lymphocytes (4-6), appear to be potential targets of fucoidan. For example, our previous study suggested that fucoidan promoted the maturation of human monocyte-derived DCs, altered the expression of cytokines and co-stimulatory molecules, and drove their differentiation toward the Th1-polarization phenotype (4). The modulation of immune cells by fucoidan provides various properties for its clinical use.

Macrophages serve important roles in eliciting and modulating the immune response (7). As the biggest population of innate immunocytes in tissue, macrophages recognize dangerous signals, and present them to T cells or B cells to initiate adaptive immunity. Additionally, macrophages regulate the function of adaptive immunity through cell-to-cell interaction or fluid-phase modulation, via cytokines, chemokines, reactive radicals and nitric oxide (8). Therefore, the biological activities of macrophages, including migration, phagocytosis and secretion of cytokines, are critical to the outcome of an immune response. Notably, macrophage subsets differ distinctly in these biological properties and their functions range from inflammatory to anti-inflammatory (9). In addition to diversity, plasticity is another characteristic of macrophages. This means that they can be phenotypically and functionally polarized to distinct subsets responding to microenvironmental signals or exogenous supplements $(10,11)$.

Fucoidan is recognized as a ligand of macrophage scavenger receptors (12), therefore macrophages may be a primary target in the immunomodulatory effects of fucoidan. However, although previous studies have reported the effects of fucoidan on various properties of macrophages, including tumoricidal activity, phagocytosis and cytokine production (13-15), controversy remains in these observations. For example, although previous results suggested that fucoidan promoted pro-inflammatory cytokine production in monocytes/macrophages $(12,16,17)$, a previous study demonstrated attenuating effects of a low-molecular-weight fucoidan on pro-inflammatory cytokine production, including interleukin-1 (IL-1) 
and tumor necrosis factor- $\alpha$ (TNF- $\alpha$ ), in a dose-dependent manner from 1-100 $\mu \mathrm{g} / \mathrm{ml}$ (18). Additionally, fucoidan exhibited bifunctional effects on another inflammatory mediator, inducible nitric oxide synthase (iNOS) (19). Specifically, a low concentration of fucoidan $(10 \mu \mathrm{g} / \mathrm{ml})$ increased basal iNOS levels in macrophages, while higher fucoidan concentrations (100 or $300 \mu \mathrm{g} / \mathrm{ml}$ ) suppressed induction of iNOS expression by lipopolysaccharide (LPS). How fucoidan modulates macrophage functions and how the inconsistency occurs is not clear. Therefore, further investigation of the effects of fucoidan on macrophages would improve the understanding of its immunomodulatory property.

Colony-stimulating factor-1 (CSF-1) is a primary regulator of the proliferation, survival and function of macrophages (20). Exogenous CSF-1 induced monocytes/macrophages to express various cytokines, including TNF- $\alpha$ and IL-1 $\beta(21,22)$. In addition, CSF-1-treated monocytes/macrophages exhibited migratory changes due to altered chemokine receptor expression (23-25). However, to the best of our knowledge, no previous studies have investigated the role of CSF-1 in the modulation of macrophages by fucoidan. The present study investigated the effect of fucoidan on pro-inflammatory cytokine production and on the migratory properties of THP-1-derived macrophages, and aimed to verify the role of CSF-1 in the modulatory function of fucoidan.

\section{Materials and methods}

Reagents and antibodies. Recombinant human CSF-1 (rhCSF-1) and interferon- $\gamma($ IFN- $\gamma)$ were purchased from R\&D Systems, Inc. (Minneapolis, MN, USA). LPS and phorbol 12-myristate 13-acetate (PMA) were obtained from Sigma-Aldrich (Merck Millipore, Darmstadt, Germany). Monoclonal anti-human M-CSF neutralizing antibodies (NAb; cat. no. AF216; $100 \mathrm{ng} / \mathrm{ml}$ ) were obtained from R\&D Systems, Inc.

Preparation of fucoidan. Fucoidan purified from $F$. vesiculosus was purchased from Sigma-Aldrich (Merck Millipore) and dissolved in PBS. The potential contamination of endotoxin in fucoidan was detected by QCL-1000 ${ }^{\circledR}$ Chromogenic LAL end-point assay (Lonza Walkersville, Inc., Walkersville, MD, USA) according to the manufacturer's manual. The detection limit of the kit was $0.1 \mathrm{EU} / \mathrm{ml}$. The endotoxin level of $100 \mu \mathrm{g} / \mathrm{ml}$ fucoidan preparation was $<0.1 \mathrm{EU} / \mathrm{ml}$.

Cell culture and generation of THP-1-derived macrophages. THP-1 human acute monocytic leukemia cell line was obtained from the American Type Culture Collection (Manassas, VA, USA). The cells were cultured in RPMI 1640 medium, (Gibco; Thermo Fisher Scientific, Inc., Waltham, MA, USA) supplemented with heat-incubated $10 \%$ fetal bovine serum (FBS; Gibco; Thermo Fisher Scientific, Inc.), $100 \mathrm{U} / \mathrm{ml}$ penicillin, and $100 \mu \mathrm{g} / \mathrm{ml}$ streptomycin at $37^{\circ} \mathrm{C}$ in an incubator with $95 \%$ air and $5 \% \mathrm{CO}_{2}$.

Generation of THP-1-derived macrophages was performed as previously described (26). Briefly, $1 \times 10^{6}$ THP-1 cells were treated with $100 \mathrm{ng} / \mathrm{ml}$ PMA for $48 \mathrm{~h}$. To generate M1-polarized THP-1 macrophages, THP-1 cells were cultured with $100 \mathrm{ng} / \mathrm{ml}$ PMA for $6 \mathrm{~h}$ and then $100 \mathrm{ng} / \mathrm{ml} \mathrm{LPS} \mathrm{and}$ $20 \mathrm{ng} / \mathrm{ml}$ IFN- $\gamma$ were added for $42 \mathrm{~h}$. In certain cases, fucoidan
$(100 \mu \mathrm{g} / \mathrm{ml})$, rhCSF-1 (100 ng/ml) or CSF-1 NAb $(2 \mu \mathrm{g} / \mathrm{ml})$ was added along with LPS and IFN- $\gamma$ for $42 \mathrm{~h}$.

Cell Counting kit-8 (CCK-8) assay for cell viability. Cell viability was measured by CCK-8 (Wuhan Boster Biological Technology, Ltd., Wuhan, China). Briefly, THP-1 cells (1x10 $/$ well) were seeded into 96 -well plates along with PMA $(100 \mathrm{ng} / \mathrm{ml})$ for $6 \mathrm{~h}$ and then various concentrations of fucoidan (50, 100 and $200 \mu \mathrm{g} / \mathrm{ml})$ were supplemented into the wells. Cell proliferation was measured after different periods of time, ranging from 6 to $72 \mathrm{~h}$, using the CCK-8 assay according to the manufacturer's instructions.

Cell migration assay. THP-1-derived macrophages were cultured in a 6-well plate, then collected and resuspended in serum-free RPMI 1640 medium at a density of $1 \times 10^{6} / \mathrm{ml}$. Then, $100 \mu 1$ serum-free medium ( $1 \times 10^{5}$ cells) was added into the upper compartment of Transwell inserts (24-well plate, $8 \mu \mathrm{m}$ pores; BD Biosciences, Franklin Lakes, NJ, USA). RPMI 1640 medium $(600 \mu \mathrm{l})$ containing $10 \%$ FBS was added into the lower chamber of the Transwell plate. After incubating at $37^{\circ} \mathrm{C}$ in $5 \% \mathrm{CO}_{2}$ for $12 \mathrm{~h}$, the migrated cells on the lower surface of the filter were fixed by $10 \%$ formalin for $15 \mathrm{~min}$ at room temperature and stained with eosin. Five random fields of each well were imaged using a light microscope (magnification, x100), and cell numbers were counted.

Reverse transcription-quantitative polymerase chain reaction $(R T-q P C R)$. Total RNA from THP-1-derived macrophages was extracted using TRIzol reagent (Invitrogen; Thermo Fisher Scientific, Inc., Waltham, MA, USA), and the cDNA was synthesized by reverse transcription. Total RNA $(1.0 \mu \mathrm{g})$ was transcribed into cDNA with oligo dT16 primers and Moloney murine leukemia virus reverse transcriptase according to the manufacturer's instructions (Invitrogen; Thermo Fisher Scientific, Inc.). GAPDH was used as an internal control. The primers for TNF- $\alpha$, IL-1 $\beta$, IL-6, CSF-1 and GAPDH are listed in Table I. The reaction mixture, including $5 \mu 12 \mathrm{X} \mathrm{SYBR}{ }^{\circledR}$ Green qPCR Master mix (Thermo Fisher Scientific, Inc.), $1 \mu \mathrm{l}$ forward primer, $1 \mu \mathrm{l}$ reverse primer, $1 \mu \mathrm{l} \mathrm{cDNA}$ and $2 \mu \mathrm{l}$ double distilled $\mathrm{H}_{2} \mathrm{O}$, was incubated at $94^{\circ} \mathrm{C}$ for $30 \mathrm{sec}, 60^{\circ} \mathrm{C}$ for $30 \mathrm{sec}$ and $72^{\circ} \mathrm{C}$ for $45 \mathrm{sec}$ for 30 cycles (LightCycler 2.0; Roche Diagnostics GmbH, Mannheim, Germany). The mRNA level of each sample was measured by the $2^{-\Delta \Delta \mathrm{Cq}}$ method (27).

Enzyme-linked immunosorbent assay (ELISA). THP-1-derived macrophages were generated and cultured in complete medium for another $24 \mathrm{~h}$. Then, culture supernatants were collected. TNF- $\alpha$ (cat. no. DTA00C), IL-1 $\beta$ (cat. no. DLB50), IL-6 (cat. no. D6050) and CSF-1 (cat. no. DMC00) concentrations in culture supernatants were determined by ELISA kits (R\&D Systems, Inc.), according to the manufacturer's instructions.

Statistical analysis. Data were primarily presented as the mean + standard deviation. The SPSS software package (version 13.0; SPSS, Inc., Chicago, IL, USA) was used for all statistical analysis. The distribution of the samples was determined via Kolmogorov-Smirnov test. The results of experiments were analyzed by unpaired $t$-test or one-way 
Table I. Primer sequences for reverse transcription-quantitative polymerase chain reaction.

\begin{tabular}{|c|c|}
\hline Gene nam & Primer sequences \\
\hline TNF- $\alpha$ & $\begin{array}{l}\text { F: 5'-ATG AGC ACT GAA AGC ATG ATC C-3' } \\
\text { R: 5'-GAG GGC TGA TTA GAG AGA GGT C-3' }\end{array}$ \\
\hline IL-1 $\beta$ & $\begin{array}{l}\text { F: 5'-TGA TGG CTT ATT ACA GTG GCA ATG-3' } \\
\text { R: 5'-GTA GTG GTG GTC GGA GAT TCG-3' }\end{array}$ \\
\hline IL-6 & $\begin{array}{l}\text { F: 5'-CAC ACA GAC AGC CAC TCA CC-3' } \\
\text { R: 5'-GCT CTG GCT TGT TCC TCA CT-3' }\end{array}$ \\
\hline CSF-1 & $\begin{array}{l}\text { F: 5'-GGA GAC CTC GTG CCA AAT TA-3' } \\
\text { R: 5'-GGC CTT GTC ATG CTC TTC AT-3' }\end{array}$ \\
\hline GAPDH & $\begin{array}{l}\text { F: 5'-GGT GGT CTC CTC TGA CTT CAA CAG-3' } \\
\text { R: 5'-GTT GCT GTA GCC AAA TTC GTT GT-3' }\end{array}$ \\
\hline
\end{tabular}

TNF- $\alpha$, tumor necrosis factor- $\alpha$; IL, interleukin; CSF, colony-stimulating factor; $\mathrm{F}$, forward; $\mathrm{R}$, reverse.

analysis of variance wherever appropriate. Tukey post-hoc comparison was performed when statistical significance was detected between datasets. $\mathrm{P}<0.05$ was considered to indicate a statistically significant difference.

\section{Results}

Fucoidan modifies the morphology and migration activity of THP-1-derived macrophages. THP-1 human acute monocytic leukemia cell line is a widely-used model for monocyte/macrophage differentiation (28). PMA, an agonist of protein kinase $\mathrm{C}$, induces the THP-1 cells to acquire a macrophage-like characteristic, which can be distinguished by morphology (29). As demonstrated in Fig. 1A, the PMA-treated THP-1 cells became attached and adopted an amoeboid morphology, exhibiting similar morphology to primary human macrophages. Therefore, this study referred to them as THP-1-derived macrophages. When LPS $(100 \mathrm{ng} / \mathrm{ml})$ and IFN- $\gamma(20 \mathrm{ng} / \mathrm{ml})$ were added for $42 \mathrm{~h}$ alongside PMA stimulation, the morphology of THP-1-derived macrophages was markedly changed from a round/spindle shape to a dendritic-like shape with large filopodia, resembling the classically activated M1 macrophages (Fig. 1A).

When fucoidan alone or combined with LPS and IFN- $\gamma$ was added during the differentiation of THP-1-derived macrophages, the morphology was modified. Specifically, fucoidan increased the percentage of rounded-shaped cells in the group stimulated by PMA only. The morphology of the LPS and IFN- $\gamma$ stimulated group adopt striking changes compared with the PMA only treated group, with loss of filopodia and acquisition of amoeboid-like morphology (Fig. 1A).

The morphology of macrophages is associated with their migratory activity (30). As fucoidan modulated the morphology of THP-1-derived macrophages, the effect of fucoidan on their migratory properties was analyzed using a Transwell assay. The result indicated that LPS and IFN- $\gamma$ increased the number of migrated THP-1-derived macrophages compared with untreated cells $(\mathrm{P}=0.0039)$. Notably, compared with groups that did not receive fucoidan, when treated by fucoidan, there were significant decreases in the number of migrated macrophages in the LPS/IFN- $\gamma$-stimulated $(\mathrm{P}=0.0161)$ and unstimulated $(\mathrm{P}=0.0342)$ groups (Fig. 1B).

It was also examined whether the effects of fucoidan on macrophage migration was dependent on its concentration. For this purpose, THP-1-derived macrophages were treated with different concentrations of fucoidan from 10-200 $\mu \mathrm{g} / \mathrm{ml}$ during the differentiation process, and their migratory properties were measured. Fig. $1 \mathrm{C}$ demonstrates that the attenuation of fucoidan on migration was dose-dependent, as its effects became significant compared with $0 \mu \mathrm{g} / \mathrm{ml}$ fucoidan at a concentration of $100 \mu \mathrm{g} / \mathrm{ml}$ in the unstimulated control $(\mathrm{P}=0.0135)$ and $\mathrm{LPS} / \mathrm{IFN}-\gamma$ treated groups $(\mathrm{P}=0.0171)$, and no significant differences in migrated cell numbers were detected between 100 and $200 \mu \mathrm{g} / \mathrm{ml}$.

To determine whether fucoidan exhibited cytotoxic effects, THP-1 cells were treated with various concentrations of fucoidan $(50,100$ and $200 \mu \mathrm{g} / \mathrm{ml})$ for different periods of time in the presence of PMA $(100 \mathrm{ng} / \mathrm{ml})$, and then cell viability was determined by using the CCK- 8 assay. Fucoidan did not exhibit significant cytotoxic effects on THP-1-derived macrophages up to $72 \mathrm{~h}$ of incubation, at a concentration of $200 \mu \mathrm{g} / \mathrm{ml}$ (data not shown). The cell proliferation curve of fucoidan at a concentration of $100 \mu \mathrm{g} / \mathrm{ml}$ is shown in Fig. 2.

Fucoidan affects cytokine production of THP-1-derived macrophages. It was also investigated whether fucoidan modified the cytokine production in THP-1-derived macrophages. Consistent with previous studies (28), LPS and IFN- $\gamma$ treatment increased pro-inflammatory cytokine production, including TNF- $\alpha$, IL- $1 \beta$ and IL- 6 , compared with unstimulated cells (Fig. 3A). Notably, when fucoidan was added, cytokine transcription and secretion were decreased compared with untreated cells. Additionally, fucoidan also reversed the augmented effect of LPS and IFN- $\gamma$ on the cytokine production of THP-1-derived macrophages (Fig. 3A).

CSF-1 is an important regulator associated with the biological behavior of macrophages, promoting their proliferation, migration and cytokine production (20). Therefore the current study also investigated whether fucoidan affected the production of CSF-1 in THP-1-derived macrophages. The results indicated that THP-1-derived macrophages produce CSF-1 ( $2 \mathrm{ng} / 1 \mathrm{x} 10^{6}$ cells over $\left.24 \mathrm{~h}\right)$. LPS and IFN- $\gamma$ treatment marginally, but not significantly, decreased CSF-1 transcription, and this attenuation was not observed at the secretory level (Fig. 3B). Notably, fucoidan significantly attenuated CSF-1 transcription and CSF-1 secretion in LPS/IFN- $\gamma$-treated and untreated groups $(\mathrm{P}<0.01$; Fig. 3B).

The effect of exposure length and the concentration of fucoidan on cytokine production were also examined. Inhibitory effects of fucoidan on CSF-1 transcription occurred $6 \mathrm{~h}$ after $100 \mu \mathrm{g} / \mathrm{ml}$ of fucoidan was added into the culture medium (12 h, P=0.0057; Fig. 4A). Additionally, fucoidan decreased CSF-1 secretion in a dose-dependent manner, with the CSF-1 levels significantly decreased by $50 \mu \mathrm{g} / \mathrm{ml}$ fucoidan compared with $0 \mu \mathrm{g} / \mathrm{ml}(\mathrm{P}=0.0061)$, and a more significant decrease was observed at a concentration of $100 \mu \mathrm{g} / \mathrm{ml}$ $(\mathrm{P}=0.0032$; Fig. 4B). Dose-dependent effects of fucoidan on the secretion of TNF- $\alpha$ and IL-1 $\beta$ were also observed (data not shown). 
A

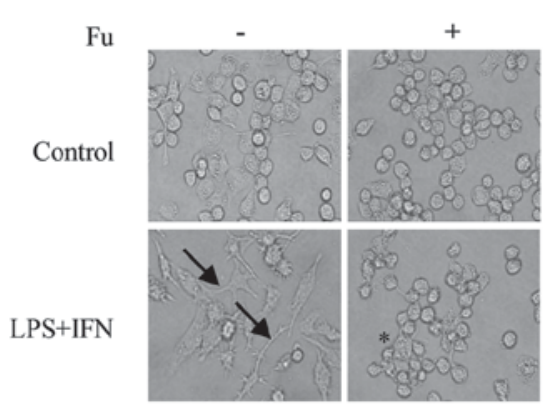

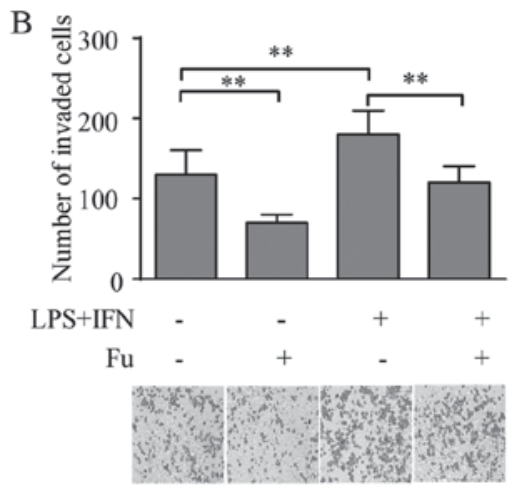

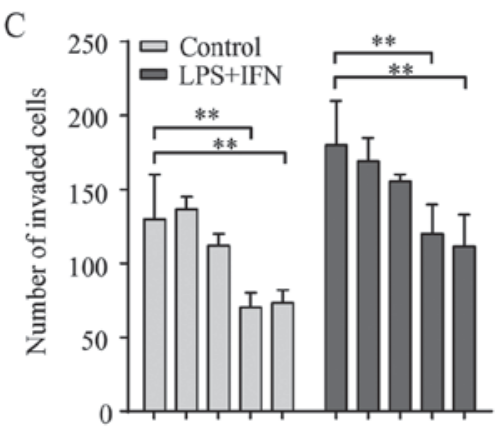

Fu $(\mu \mathrm{g} / \mathrm{ml}) 0105010020001050100200$

Figure 1. Fu modifies the morphology and migration of THP-1-derived macrophages. (A) Morphology of THP-1-derived macrophages was observed by light microscopy. Arrow, the large filopodia of THP-1-derived macrophages treated by LPS and IFN- $\gamma$; Asterisk, the amoeboid-like morphology under the stimulation of fucoidan. (B) THP-1-derived macrophages $\left(1 \times 10^{5}\right.$ per well) following stimulation were seeded in the upper chamber of 24 -well Transwell inserts and a migration assay was performed. The number of migrated cells was the mean value of five random fields observed under a light microscope. (C) Numbers of migrated THP-1-derived macrophages treated by different concentrations of fucoidan (mean + standard deviation, $\mathrm{n}=3$; ${ }^{*} \mathrm{P}<0.05 ;{ }^{* *} \mathrm{P}<0.01$ ). Fu, fucoidan; LPS, lipopolysaccharide; IFN- $\gamma$, interferon- $\gamma$.

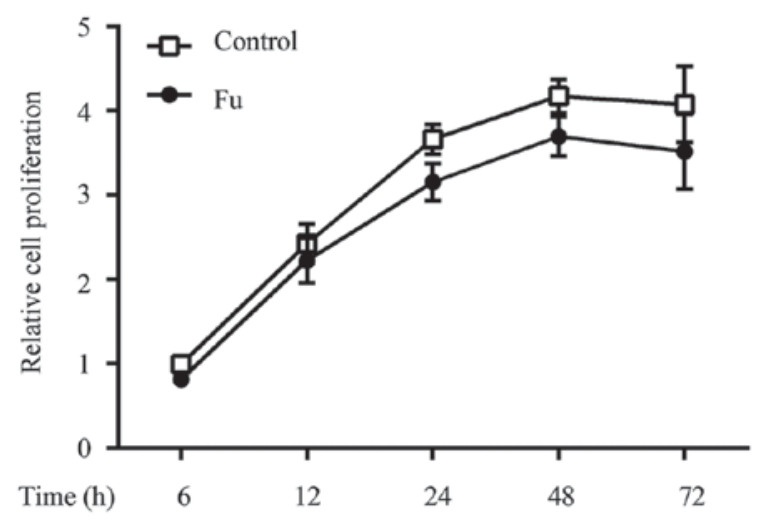

Figure 2. Fu exhibits minimal cytotoxic effects on THP-1-derived macrophages within $72 \mathrm{~h}$. Fu was supplemented into THP-1 cells after $6 \mathrm{~h}$ of phorbol 12-myristate 13-acetate for an intended period of time, and then cell proliferation was measured by Cell Counting Kit-8 assay. The proliferation curves of THP-1-derived macrophages under the treatment of no (control) or $100 \mu \mathrm{g} / \mathrm{ml} \mathrm{Fu}$ are presented (mean \pm standard deviation, $\mathrm{n}=3$ ). Fu, fucoidan.

CSF-1 is involved in the production of pro-inflammatory cytokines modified by fucoidan. Previous investigation revealed that CSF-1 promoted pro-inflammatory cytokine production in monocytes/macrophages (21). Because fucoidan significantly decreased CSF-1 secretion in THP-1-derived macrophages, it was also determined whether the reduced CSF-1 level was responsible for the attenuation of pro-inflammatory cytokine expression. Initially, CSF-1 NAb $(2 \mu \mathrm{g} / \mathrm{ml})$ was added with LPS and IFN- $\gamma$ during the differentiation of THP-1-derived macrophages. PCR and ELISA results demonstrated that blocking endogenous CSF-1 expression significantly decreased pro-inflammatory cytokine production in macrophages compared with the control groups $(\mathrm{P}<0.05$; Fig. 5A). Additionally, rhCSF-1 $(100 \mathrm{ng} / \mathrm{ml})$ was added with/without fucoidan to THP-1-derived macrophages in the presence of LPS and IFN- $\gamma$. RT-qPCR results revealed that rhCSF-1 significantly increased the transcription of TNF- $\alpha$, IL- $1 \beta$ and IL- 6 with or without fucoidan treatment compared with no rhCSF-1 treatment $(\mathrm{P}<0.01 ;$ Fig. 5B). The effects of rhCSF-1 on cytokine secretion was somewhat different from the transcription level, as rhCSF-1 did not significantly alter the cytokine secretion of THP-1-derived macrophages without fucoidan treatment, however, did reverse the attenuating effect of fucoidan on the secretion of these cytokines ( $\mathrm{P}<0.05$; Fig. 5C).

CSF-1 is associated with the migratory activity of THP-1-derived macrophages modified by fucoidan. The current study also explored whether the altered expression of CSF-1 was responsible for the migration of THP-1-derived macrophages. Exogenous CSF-1 treatment did not cause morphological change in macrophages, in either fucoidan treated or untreated groups (Fig. 6A). However, when stimulated with rhCSF-1, the migratory activity of THP-1-derived macrophages was significantly increased compared with the control $(\mathrm{P}<0.01$; Fig. 6B). More importantly, the suppression of migration by fucoidan was also reversed by rhCSF-1 ( $\mathrm{P}<0.01$; Fig. 6B).

\section{Discussion}

The present study analyzed the effects of fucoidan on the migration and cytokine production of THP-1-derived macrophages, and aimed to examine the mechanisms involved in this modulation. The current study demonstrated that fucoidan-treated THP-1-derived macrophages exhibited morphological changes, reduced migration and pro-inflammatory cytokine production. In addition, fucoidan also decreased CSF-1 production and exogenous CSF-1 reversed the attenuating effect of fucoidan. Therefore, the results indicate that fucoidan reduced the pro-inflammatory capacity of THP-1-derived macrophages. This effect may be modulated by CSF-1 expression.

THP-1-derived macrophages exhibit specialized morphological and functional characteristics in response to exogenous stimulations. Initially, morphological changes in macrophages were observed when fucoidan was added; filopodia formation was decreased and macrophages adopted a more amoeboid-like morphology. Additionally, fucoidan reduced the migratory properties of THP-1-derived macrophages, with/without LPS and IFN- $\gamma$ stimulation, however, the mechanisms of modulation remained unresolved. Cell migratory activity is dependent on various factors, for example, 
A
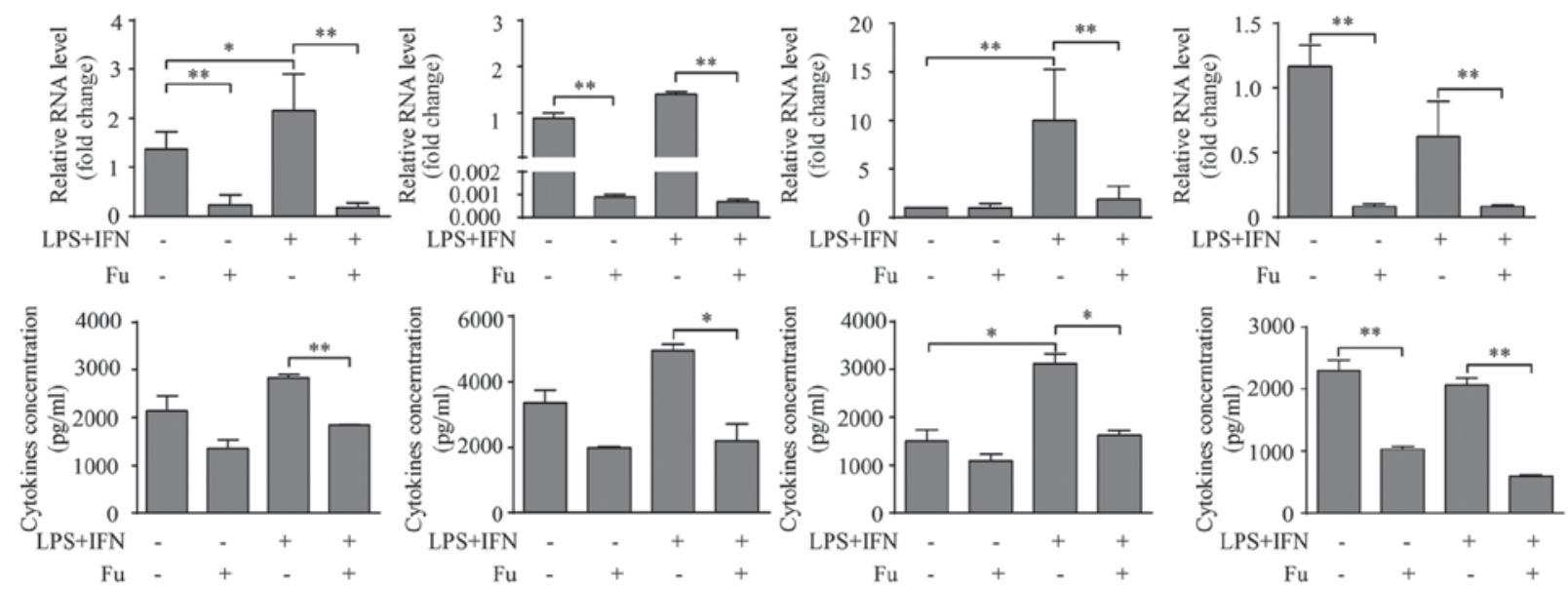

Figure 3. Fu modifies the secretory activity of THP-1-derived macrophages. (A) The transcription and secretion of pro-inflammatory cytokines, TNF- $\alpha$, IL-1 $\beta$ and IL-6 with or without Fu treatment, was measured by RT-qPCR (upper) and ELISA (lower). (B) The transcription (upper) and secretion (lower) of CSF-1 was measured. The RT-qPCR data were normalized to the control and presented as the fold change. Each bar represents the mean + standard deviation ( $\mathrm{n}=3$, $\left.{ }^{*} \mathrm{P}<0.05,{ }^{* *} \mathrm{P}<0.01\right)$. Fu, fucoidan; TNF- $\alpha$, tumor necrosis factor- $\alpha$; IL, interleukin; LPS, lipopolysaccharide; IFN, interferon; CSF, colony-stimulating factor; RT-qPCR, reverse transcription-quantitative polymerase chain reaction.

A

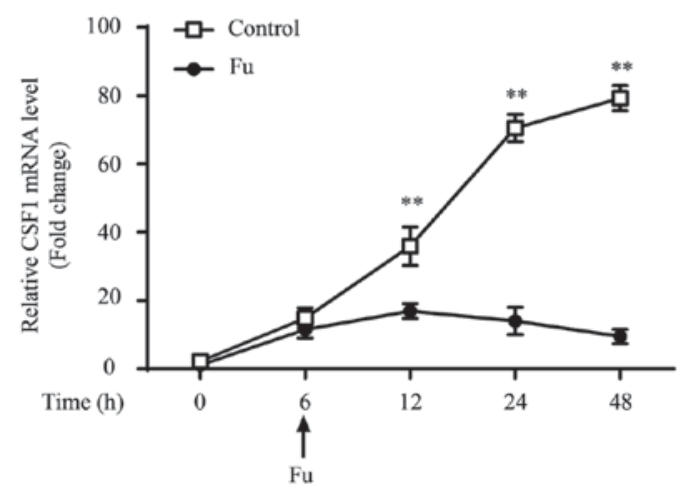

B

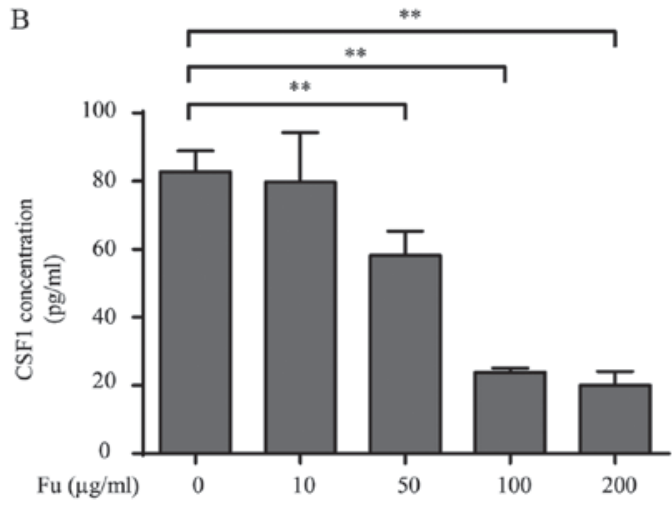

Figure 4. Time and dose-dependent effects of Fu on CSF-1 expression. (A) Fu (100 $\mu \mathrm{g} / \mathrm{ml})$ was added after $6 \mathrm{~h}$ of phorbol 12 -myristate 13 -acetate (100 ng/ml) stimulation. THP-1-derived macrophages were collected at different time points and CSF-1 transcription was measured by RT-qPCR. The RT-qPCR data were normalized to the control group at $0 \mathrm{~h}$ and presented as the fold change. The transcription levels at the same time point between the control and fucoidan-treated group were compared. (B) Different concentrations of fucoidan were added to THP-1-derived macrophages for $42 \mathrm{~h}$. CSF-1 concentration in supernatant was measured by enzyme-linked immunosorbent assay (mean \pm standard deviation, $\mathrm{n}=3 ;{ }^{*} \mathrm{P}<0.05,{ }^{* *} \mathrm{P}<0.01$ ). Fu, fucoidan; $\mathrm{CSF}$, colony-stimulating factor; RT-qPCR, reverse transcription-quantitative polymerase chain reaction.

chemokine secretion, integrin expression, expression of matrix metalloproteinases (MMPs) and cytoskeleton organization (31). Our previous study demonstrated that fucoidan increased TNF- $\alpha$-induced proMMP-9 in human monocyte U937 cells, suggesting that fucoidan regulates MMPs expression (32). In addition, cytoskeleton arrangement regulates the type of cell movement, and also the migratory properties (33). Because fucoidan significantly altered the morphology of THP-1-derived macrophages, there was a possibility that fucoidan regulated macrophage migration by reorganizing the cytoskeleton. Another previous study reported that fucoidan-treated MDA-MB-231 breast cancer cells also adopt morphological changes similar to LPS/IFN- $\gamma$-treated macrophages, loss of cell spreading occurred with cytoskeleton reorganization and depletion of vinculin (34). Whether similar mechanisms occur in THP-1-derived macrophages requires further investigation.

In addition to the migratory properties, fucoidan also greatly attenuated pro-inflammatory cytokine production, including TNF- $\alpha$, IL- $1 \beta$ and IL- 6 . This attenuation by fucoidan was significant, and the stimulating effects of LPS and IFN- $\gamma$ were almost fully reversed. The effects of fucoidan on cytokine production by macrophages remains controversial, as certain studies have reported that fucoidan induces pro-inflammatory cytokine production, including TNF- $\alpha$ and IL-1, in macrophages (12), while others demonstrated an attenuating effect (18). How this paradox exists remains unclear but is acceptable, as the biological activities of fucoidan are different depending on seaweed species or the structural and compositional characteristics (35). The 

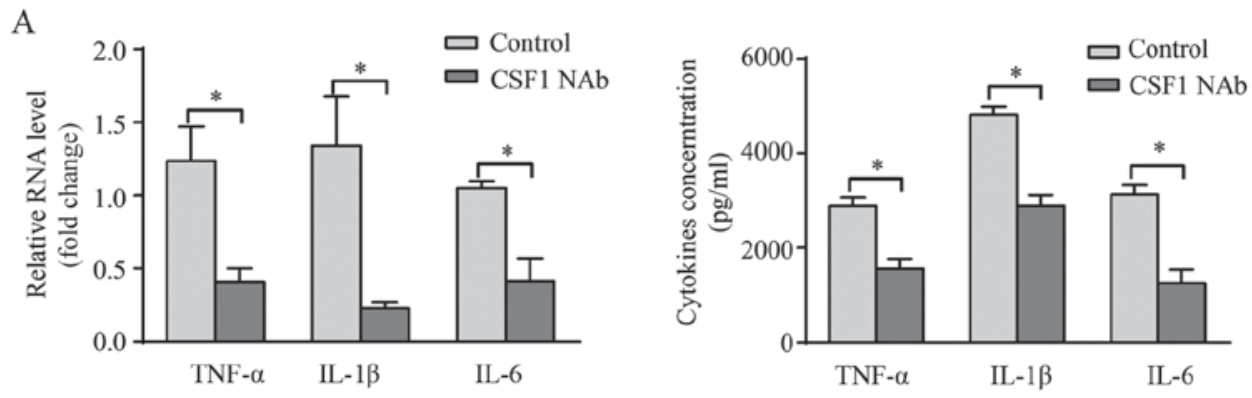

B

TNF- $\alpha$
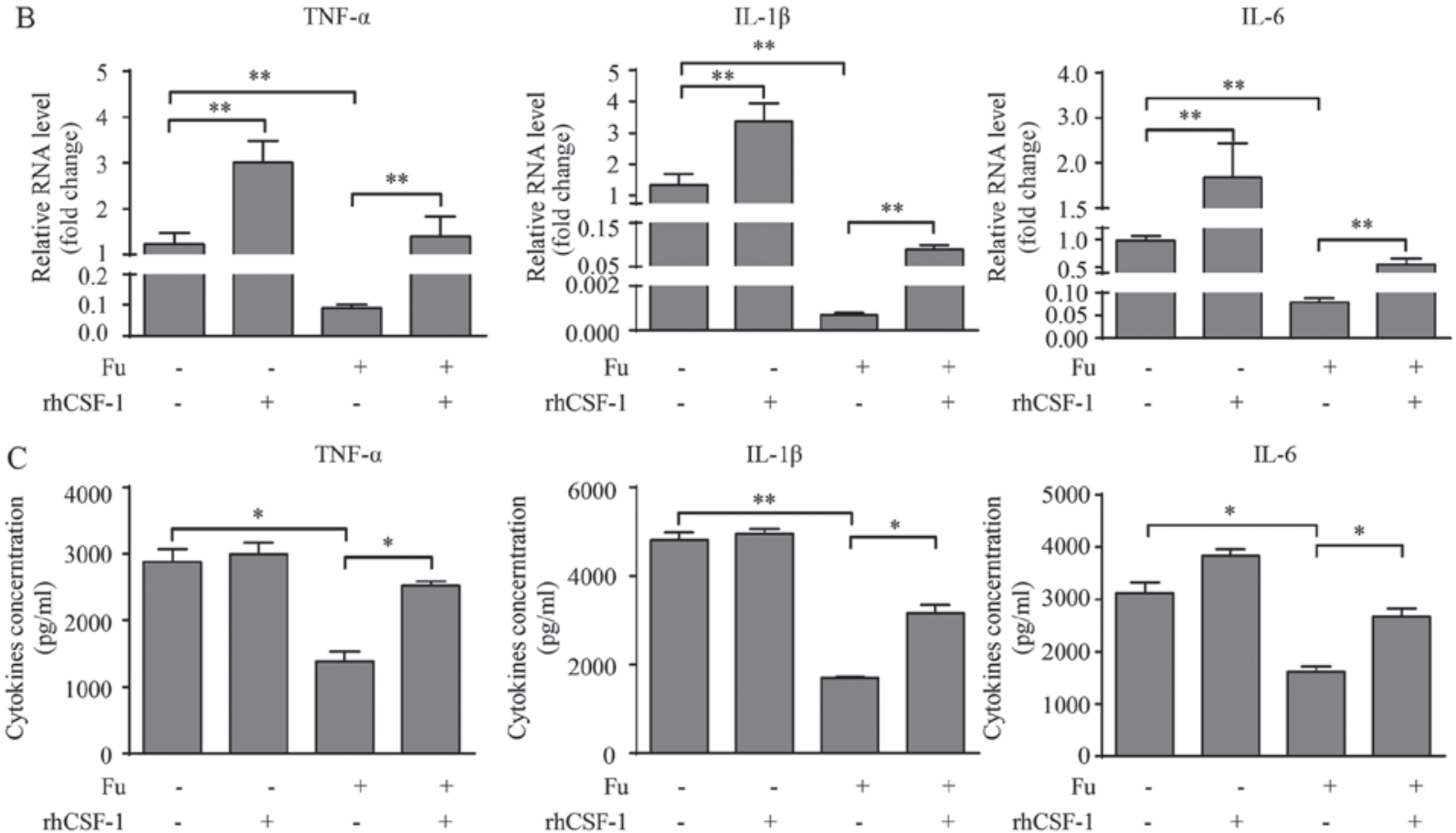

Figure 5. CSF-1 is involved in Fu-modulated cytokine production of THP-1-derived macrophages. (A) Transcription and secretion of TNF- $\alpha$, IL-1 $\beta$ and IL-6 with or without CSF-1 NAb were measured by RT-qPCR (left) and ELISA (right). rhCSF-1 and/or Fu were added to the THP-1-derived macrophages. (B) Transcription and (C) secretion of TNF- $\alpha$, IL-1 $\beta$ and IL-6 were measured. The RT-qPCR data were normalized to the control and shown as the fold change. Each bar represents the mean + standard deviation $\left(n=3,{ }^{*} \mathrm{P}<0.05,{ }^{* *} \mathrm{P}<0.01\right)$. CSF, colony-stimulating factor; Fu, fucoidan; TNF- $\alpha$, tumor necrosis factor- $\alpha$; IL, interleukin; NAb, neutralizing antibody; RT-qPCR, reverse transcription-quantitative polymerase chain reaction; rhCSF, recombinant human colony-stimulating factor.

$\mathrm{Fu}$
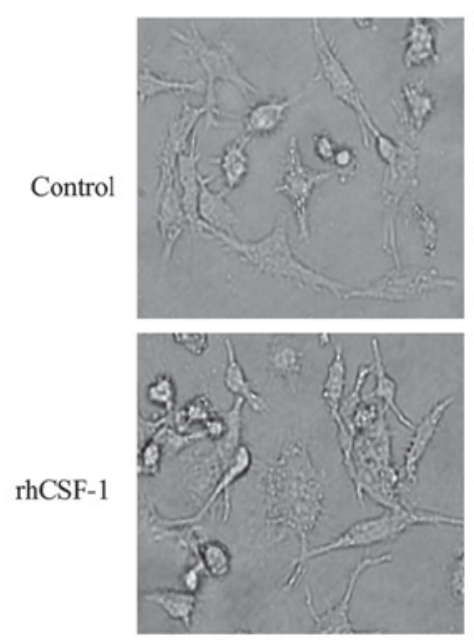
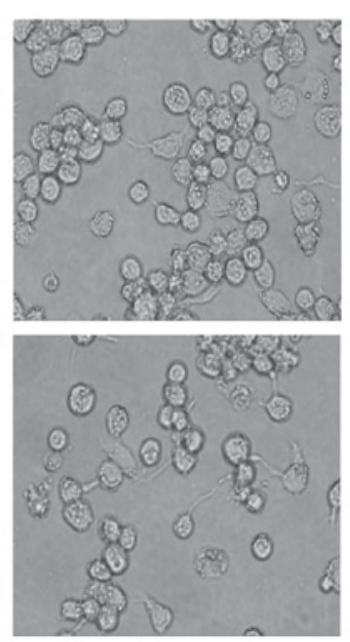
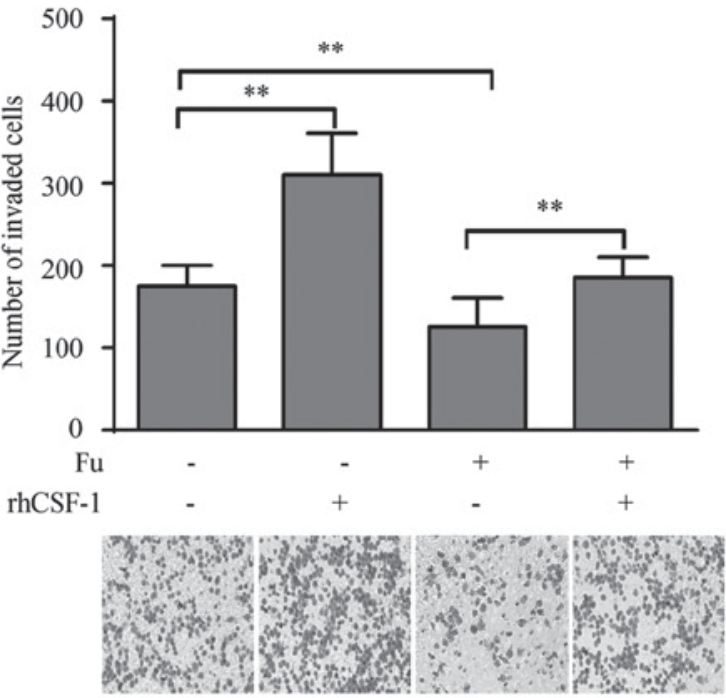

Figure 6. rhCSF-1 reverses Fu-modulated migration of THP-1-derived macrophages. Morphology of THP-1-derived macrophages was observed by light microscopy. The migration was measured by Transwell assay. Number of migrated cells was the mean value of five random fields under a light microscope (mean + standard deviation, $\mathrm{n}=3$ ). rhCSF, recombinant human colony-stimulating factor; Fu, fucoidan. 
findings of the current study enriched the evidence for the immunomodulatory effects of fucoidan, particularly in macrophages. Furthermore, as the bioactive functions of fucoidan vary, which is demonstrated by the present and previous studies, a full understanding of the functions and structural molecular features of fucoidan is required prior to its clinical application.

CSF-1 is known as a primary regulator of cytotoxicity, chemotaxis and cytokine production in monocytes/macrophages, therefore, it is closely associated with their inflammatory properties $(21,22)$. Macrophages are important producers and targets of CSF-1. The present study demonstrated that THP-1-derived macrophages produced high levels of CSF-1, and LPS and IFN- $\gamma$ marginally, but not significantly, decreased CSF-1 production. Importantly, fucoidan greatly attenuated CSF-1 transcription and secretion in LPS and IFN- $\gamma$ treated/untreated groups. To the best of our knowledge, this is the first time that fucoidan has been reported to regulate CSF-1 production in macrophages. In order to determine whether decreased CSF-1 was involved in the migration and cytokine production of macrophages, two experiments were we performed. CSF-1 NAb was added during the differentiation process to block basic CSF-1 production of macrophages. Pro-inflammatory cytokine production, which was stimulated by LPS and IFN- $\gamma$, was significantly decreased following blocking of CSF-1. Recombinant human CSF-1 (100 ng/ml) was then added to THP-1-derived macrophages with/without fucoidan treatment. rhCSF-1 treatment almost completely reversed the attenuation of pro-inflammatory cytokine production by fucoidan, particularly TNF- $\alpha$ and IL-6. Furthermore, THP-1-derived macrophages regained their migratory activity following rhCSF-1 treatment. described above experiments have limitations, as it is difficult to determine whether rhCSF-1 has similar structural traits, decomposition time and biological activities to endogenous CSF-1 secreted by macrophages. Nevertheless, the present study demonstrated that fucoidan attenuated CSF-1 production in THP-1-derived macrophages, and CSF-1 concentration was positively associated with pro-inflammatory cytokine production and migration. Therefore, fucoidan-modulated CSF-1 production may be a potential mechanism that mediates biological changes observed in this study.

In conclusion, the current study demonstrated that fucoidan attenuated the migration and pro-inflammatory cytokine production of THP-1-derived macrophages. Fucoidan-modulated CSF-1 production may be responsible for this attenuation. These results provide novel insights into the biological properties of fucoidan as an immunomodulatory agent, particularly its regulatory effects on macrophages.

\section{Acknowledgements}

This work was supported by the National Natural Science Foundation of China (grant nos. 31300752, 31470885, 31270971, 81072406 and 81300510).

\section{References}

1. Chizhov AO, Dell A, Morris HR, Haslam SM, McDowell RA, Shashkov AS, Nifant'ev NE, Khatuntseva EA and Usov AI: A study of fucoidan from the brown seaweed Chorda filum. Carbohydr Res 320: 108-119, 1999.
2. Bilan MI, Grachev AA, Ustuzhanina NE, Shashkov AS, Nifantiev NE and Usov AI: Structure of a fucoidan from the brown seaweed Fucus evanescens C. Ag. Carbohydr Res 337: 719-730, 2002.

3. Sang VT and Kim SJ: Fucoidans as a natural bioactive ingredient for functional foods. J Function Foods 5: 16-27, 2013.

4. Yang M, Ma C, Sun J, Shao Q, Gao W, Zhang Y, Li Z, Xie Q, Dong $\mathrm{Z}$ and $\mathrm{Qu} \mathrm{X}$ : Fucoidan stimulation induces a functional maturation of human monocyte-derived dendritic cells. Int Immunopharmacol 8: 1754-1760, 2008.

5. Maruyama $\mathrm{H}$, Tamauchi $\mathrm{H}$, Hashimoto $\mathrm{M}$ and Nakano $\mathrm{T}$ : Antitumor activity and immune response of Mekabu fucoidan extracted from Sporophyll of Undaria pinnatifida. In Vivo 17: 245-249, 2003.

6. Oomizu S, Yanase Y, Suzuki H, Kameyoshi Y and Hide M: Fucoidan prevents $\mathrm{C}$ epsilon germline transcription and NFkappaB p52 translocation for IgE production in B cells. Biochem Biophys Res Commun 350: 501-507, 2006.

7. Epelman S, Lavine KJ and Randolph GJ: Origin and functions of tissue macrophages. Immunity 41: 21-35, 2014.

8. Fujiwara $\mathrm{N}$ and Kobayashi $\mathrm{K}$ : Macrophages in inflammation. Curr Drug Targets Inflamm Allergy 4: 281-286, 2005.

9. Mantovani A, Sozzani S, Locati M, Allavena P and Sica A: Macrophage polarization: Tumor-associated macrophages as a paradigm for polarized M2 mononuclear phagocytes. Trends Immunol 23: 549-555, 2002.

10. Biswas SK and Mantovani A: Macrophage plasticity and interaction with lymphocyte subsets: Cancer as a paradigm. Nat Immunol 11: 889-896, 2010.

11. Mantovani A, Biswas SK, Galdiero MR, Sica A and Locati M: Macrophage plasticity and polarization in tissue repair and remodelling. J Pathol 229: 176-185, 2013.

12. Hsu HY, Chiu SL, Wen MH, Chen KY and Hua KF: Ligands of macrophage scavenger receptor induce cytokine expression via differential modulation of protein kinase signaling pathways. J Biol Chem 276: 28719-28730, 2001.

13. Choi EM, Kim AJ, Kim YO and Hwang JK: Immunomodulating activity of arabinogalactan and fucoidan in vitro. J Med Food 8: 446-453, 2005

14. Kasai A, Arafuka S, Koshiba N, Takahashi D and Toshima K: Systematic synthesis of low-molecular weight fucoidan derivatives and their effect on cancer cells. Org Biomol Chem 13: 10556-10568, 2015.

15. Senthilkumar K and Kim SK: Anticancer effects of fucoidan. Adv Food Nutr Res 72: 195-213, 2014.

16. Mytar B, Woloszyn M, Macura-Biegun A, Hajto B, Ruggiero I, Piekarska B and Zembala M: Involvement of pattern recognition receptors in the induction of cytokines and reactive oxygen intermediates production by human monocytes/macrophages stimulated with tumour cells. Anticancer Res 24: 2287-2293, 2004.

17. Mytar B, Gawlicka M, Szatanek R, Woloszyn M, Ruggiero I, Piekarska B and Zembala M: Induction of intracellular cytokine production in human monocytes/macrophages stimulated with ligands of pattern recognition receptors. Inflamm Res 53: 100-106, 2004

18. Kim KJ, Yoon KY and Lee BY: Low molecular weight fucoidan from the sporophyll of Undaria pinnatifida suppresses inflammation by promoting the inhibition of mitogen-activated protein kinases and oxidative stress in RAW264.7 cells. Fitoterapia 83: 1628-1635, 2012

19. Yang JW, Yoon SY, Oh SJ, Kim SK and Kang KW: Bifunctional effects of fucoidan on the expression of inducible nitric oxide synthase. Biochem Biophys Res Commun 346: 345-350, 2006.

20. Akagawa KS: Functional heterogeneity of colony-stimulating factor-induced human monocyte-derived macrophages. Int $\mathbf{J}$ Hematol 76: 27-34, 2002.

21. Ji XH, Yao T, Qin JC, Wang SK, Wang HJ and Yao K: Interaction between M-CSF and IL-10 on productions of IL-12 and IL-18 and expressions of CD14, CD23, and CD64 by human monocytes. Acta Pharmacol Sin 25: 1361-1365, 2004.

22. Sweet MJ, Campbell CC, Sester DP, Xu D, McDonald RC, Stacey KJ, Hume DA and Liew FY: Colony-stimulating factor-1 suppresses responses to CpG DNA and expression of toll-like receptor 9 but enhances responses to lipopolysaccharide in murine macrophages. J Immunol 168: 392-399, 2002.

23. Ancuta P, Rao R, Moses A, Mehle A, Shaw SK, Luscinskas FW and Gabuzda D: Fractalkine preferentially mediates arrest and migration of CD16+ monocytes. J Exp Med 197: 1701-1707, 2003. 
24. Sierra-Filardi E, Nieto C, Domínguez-Soto A, Barroso R, Sánchez-Mateos P, Puig-Kroger A, López-Bravo M, Joven J, Ardavín C, Rodríguez-Fernández JL, et al: CCL2 shapes macrophage polarization by GM-CSF and M-CSF: Identification of CCL2/CCR2-dependent gene expression profile. J Immunol 192: 3858-3867, 2014.

25. Reimer MK, Brange $C$ and Rosendahl A: CCR8 signaling influences Toll-like receptor 4 responses in human macrophages in inflammatory diseases. Clin Vaccine Immunol 18: 2050-2059, 2011.

26. Zhao P, Gao D, Wang Q, Song B, Shao Q, Sun J, Ji C, Li X, Li P and Qu X: Response gene to complement 32 (RGC-32) expression on M2-polarized and tumor-associated macrophages is M-CSF-dependent and enhanced by tumor-derived IL-4. Cell Mol Immunol 12: 692-699, 2015

27. Livak KJ and Schmittgen TD: Analysis of relative gene expression data using real-time quantitative PCR and the 2(-Delta Delta C(T)) Method. Methods 25: 402-408, 2001.

28. Daigneault M, Preston JA, Marriott HM, Whyte MK and Dockrell DH: The identification of markers of macrophage differentiation in PMA-stimulated THP-1 cells and monocyte-derived macrophages. PloS One 5: e8668, 2010.

29. Tsuchiya S, Kobayashi Y, Goto Y, Okumura H, Nakae S, Konno T and Tada K: Induction of maturation in cultured human monocytic leukemia cells by a phorbol diester. Cancer Res 42 : 1530-1536, 1982.
30. Vogel DY, Heijnen PD, Breur M, de Vries HE, Tool AT, Amor $S$ and Dijkstra CD: Macrophages migrate in an activation-dependent manner to chemokines involved in neuroinflammation. J Neuroinflammation 11: 23, 2014.

31. Louis SF and Zahradka P: Vascular smooth muscle cell motility: From migration to invasion. Exp Clin Cardiol 15: e75-e85, 2010

32. Sun J, Feng A, Zhang Y, Sun S, Hu W, Yang M, Wei F and Qu X: Fucoidan increases TNF-alpha-induced MMP-9 secretion in monocytic cell line U937. Inflamm Res 59: 271-276, 2010.

33. Rougerie P, Miskolci V and Cox D: Generation of membrane structures during phagocytosis and chemotaxis of macrophages: Role and regulation of the actin cytoskeleton. Immunol Rev 256: 222-239, 2013

34. Liu JM, Bignon J, Haroun-Bouhedja F, Bittoun P, Vassy J, Fermandjian S, Wdzieczak-Bakala J and Boisson-Vidal C: Inhibitory effect of fucoidan on the adhesion of adenocarcinoma cells to fibronectin. Anticancer Res 25: 2129-2133, 2005.

35. Ale MT, Mikkelsen JD and Meyer AS: Important determinants for fucoidan bioactivity: A critical review of structure-function relations and extraction methods for fucose-containing sulfated polysaccharides from brown seaweeds. Marine drugs 9: 2106-2130, 2011. 